\title{
CONSTRUCTING AN INFORMATIONAL PLATFORM FOR FAMILY AND SCHOOL USING GPRS
}

\author{
Yongping Gao *, Yueshun $\mathrm{He}$ \\ School of Information Technology East China Institue of Technology, FuZhou, JiangXi, China \\ * Corresponding author, School of Information Technology East China Institue of Tech- \\ nology, FuZhou, JiangXi, China, Email:ypgao_ypgao@163.com
}

\begin{abstract}
The article is mainly about how to employ IC card and mobile phone short messages to construct a convenient and real time information communication platform in the educational field, and to quicken the transmission of educational information so as to realize benefits of communication to education. It introduces the whole schematic design, and the designs of the software mainly include Web serving software, the desktop application software, background service software, two types of machines, controlling software, short messages gateway and so on.
\end{abstract}

Keywords: schematic design, IC card, two types of machines, ActiveX controls, short message gateway

\section{INTRODUCTION}

With the advent of information age and the development of communication, there are more and more demands for information. Internet and cell phones are becoming important tools for people to obtain information and to keep in touch. Their applications can be seen in different fields, and they play an important part in these fields. The article is mainly about how to construct a multi-functional platform of short messages, sounds 
and Web by combining IC card, GSM cell phones with short messages. The platform is to realize a real time information communication and educational communication among schools, parents and pupils, and to realize the modernization of family education with elementary school information (Jinhua, 2004).

\section{THE WHOLE SCHEMATIC DESIGN}

The system consists of hardware and software. Hardware is composed of IC card terminals and IC card phones. They achieve the collection and transmission of IC card data, and accept the results and commands from upper layer software. Software is responsible for dealing with IC card data and answering the requests of hardware (Ruhong Gong, 2004) [2]. Hardware and software work together to realize the whole systematic goal and functions. The topological structure of network in the system is as the following fig. 1.

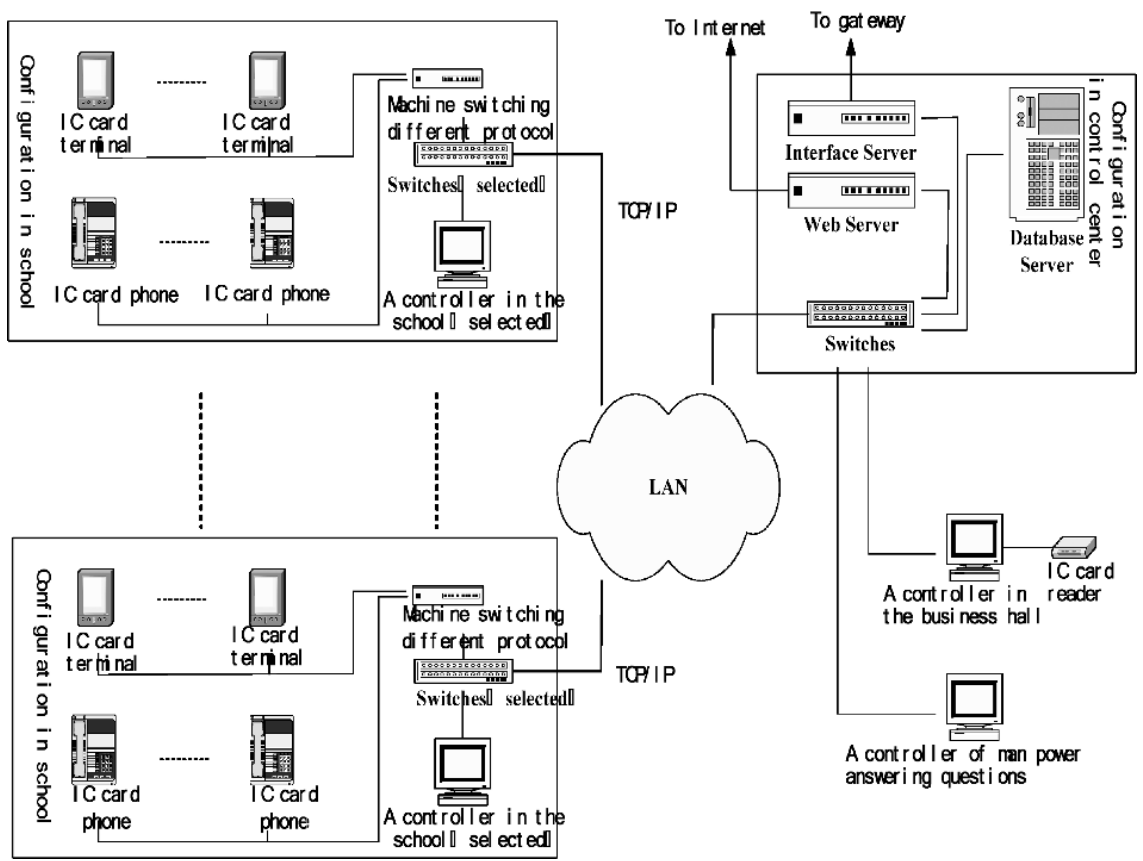

Fig. 1. Based on the GPRS about the informational platform project of family and school

Database server is connected to every IC card terminal and phone in a local network. The collection and transmission of data have been done by placing LAN lines connected with IC card terminals and phones in schools. A machine equipped with an Ethernet controller transmits the data to the database server over the LAN. The database server manages the data, and the results will be transmitted to the IC card terminal or phone. 
The functions of management interface and web server can be done by a PC. The software in the interface controller reads the message(s) data which needs to be sent to the server database and send the message(s) to parent cell phones through the short message gateway. Parents can send enquiry messages to short message gateway from their cell phones. The software will search the database and inform parents of the results via the short message gateway. There are two network cards. One is on the same subnet as the PC and the database server. The other is set as the Internet IP address and it provides web server functionality and can send message to the short messages gateway.

A controller in the business hall runs software which deals with matters such as card management and is responsible for IC cards business of all schools.

A controller of man power answering questions makes use of 1860 information service to provide terminal operation software to accept the man power service hot lines.

A controller in the school mainly runs the software for school management.

\section{THE DESIGN OF SOFTWARE STRUCTURE OF SYSTEM}

Nowadays, three-layer (multilayer) structure has become the mainstream in developing software. It is derived from traditional two-layer (Client/ Server) structure, and represents the future of enterprise applications. The software structure chart of system follows in fig. 2.

The software structure of system has some features that it is combined model B/S (Browser/Server) with model C/S (Client/Server), and that it adds business logic layer between application layer and database layer to form three-layer client/server framework (Wei Chen, 2004). The Web server answers the requests from the browser. If the requests include database operations, the Web server will switch the requests to the business logic layer, and then the business logic layer will perform the database operation and return the results to the Web server. Correspondingly, the Web server will produce and return the results to the browser. Similarly, if there are database requests from application software within the application platform, the operation will be performed by the business logic layer. In order to hide the lower communication details, the service software of the service platform needs to communicate with IC cards and the short message gateway, so it uses ActiveX Controls which provide events and methods by which the communication can be accomplished. The main design thoughts and implementation functions are as follows: 


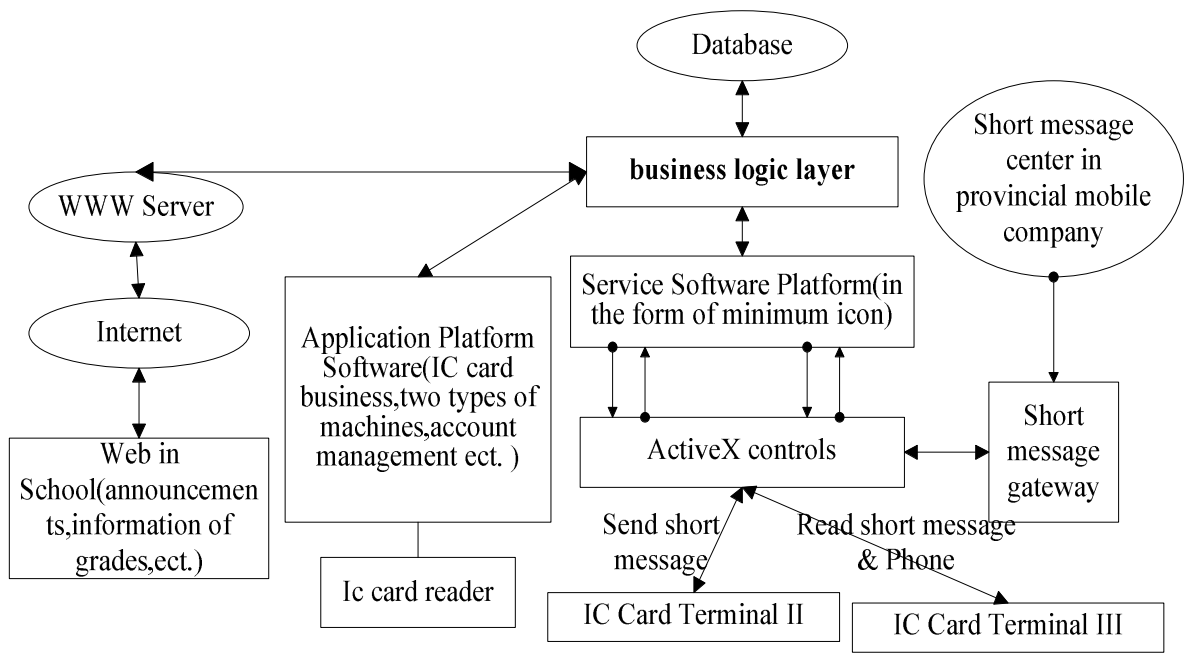

Fig. 2. The software structure chart of system

\subsection{The Design of Web Service Software}

The Web service is implemented in the Web server and the Web pages can be accessed by the browser on Internet or local network. Teachers can use their own IDs and passwords to login in and send announcements to parents of school activities, how pupils perform in school and so on. Teachers and parents can know whether pupils have arrived at school safely. They can communicate with parents online, and when their pupils have any difficulty, and they can work out a better way with parents. And parents can use pupils IDs and passwords to login in and communicate with teachers.

\subsection{The Design of Application Platform Software}

The application platform software includes IC card business management software, school management software and 1860 service software.

IC card business management software is installed on the PC in the business hall reception desk, and only the person who has the access rights can operate it. This software mainly manages IC card business transactions, information management for the IC cards, management of equipment, statistics and general system management.

School management software is installed on the PC in the schools, and it is operated by the administrator(s) within the schools. The software provides 
the following functions: binding teacher cards with pupil cards, looking into whether pupils are in school or not, and sending announcements to teachers.

1860 service software is installed in the information service platform at the mobile corporation and is used by 1860 operators. The software has the main functions of leaving messages, sending short messages between teachers and pupils when they dial man power hot lines.

\subsection{The Design of Service Software Platform}

The service software platform, in the form of minimum icon, runs on the PC which acts as database server and administrating interface. It consists of three function modules: IC card service, short messages service and accounts management.

The communication software, accepting IC card data and answering requests between every IC card terminal and phone at the schools, is the most important function of the IC card service module. After running this program, the local terminal 5009 will be opened to take over the data and demands of IC cards through the Ethernet. The system employs Command/Response communications mechanism: When a user puts an IC card in IC card terminal, the data on the IC card will be transmitted to the IC card service module. At this time, the terminal is waiting for a response from the IC card service module. If the answer is received in a certain time, the communication succeeds. Otherwise the communication fails. The state of communication can be examined in real time by this method.

The main function of the short message service module is to set up connection with the mobile short message gateway, and transmit and receive short messages to and from the short messages gateway on the Internet. The program received the messages to be sent at any time, and sends the messages one by one to the short message gateway until all have been completed, and it waits for a second, before repeating the whole process.

The account management module is to create accounts information every month, and to update the status of IC cards with the status of parent cell phones. The program can automatically produce all the accounts information of IC cards in the last day per month, and transmit this data to BOSS charging system which will deduct appointed fees from parent's cell phone account. In addition, the program downloads the updated information of parent cell phones from BOSS charging system every day, and renews data of IC cards according to different information of cell phones. 


\subsection{The Design of Two Types of Machines-the Design of IC Card Terminal and IC Card Phone}

IC card reader is to initialize IC cards and there are these kinds of readers in market.

IC card terminal is designed by us, which includes MSC, screen and circuit etc. when a user puts an IC card close to the filed of IC card reader terminal, the machine can read the data of this card, and transmit the data to the service platform software ${ }^{[4]}$.

An IC card phone has been added LCD, headphone, keyboard, GSM card on the basis of IC card terminal. Its appearance resembles a phone. Its working model is request-answer, and users can operate it using menus. It has the functions of dialing stored numbers, reading short messages from parent cell phones and sending the stored short messages.

\subsection{The Design of ActiveX Controls}

We have designed an OCX Control to accomplish the communication and transmission among IC card terminals, IC card phones and the server. The control comprises many events and methods. It deals with the primitive data and hides the details of communication in the lower layer, and provides a normal interface to the upper layer software. The main events and methods are as follows:

OnICRWRegist Event: This is called when a registration request is received from an active card in an IC Card Terminal.

OnConnect Event: This is called when a command is sent to IC Card Terminal and IC Card Terminal gives response correspondingly.

OnGetUser Event: This is called when data are received from IC card terminals.

ReqLeaveWords Event: This is used to process requests to receive short messages from the IC Card.

OnReqForRTAndCN Event: Used to retrieve phone numbers and balances from IC Card Phones

OnFeeRemainTime Event: Updates the balances on the IC Card Phone

FSInitPort Method: Initialises communication between the server and IC Card Terminal or Phone

AckICRW Method: This is used to deal with a registration by the upper software after a registration request is received from an active card in an IC Card Terminal.

ShotTSN Method: This is used by the upper layer software to switch the pupils account numbers and their names to IC card terminals or phones. 
ShowLeaveWord Method: This is used by the upper layer software to transmit total short messages, their sequence numbers and contents to IC card phones.

ShowLeaveWord Method: this is used by the upper layer software to transmit total short messages, their sequence numbers and contents to IC card phones.

\subsection{The Design of the Short Messages Gateway}

We have developed an OCX control as an interface (Jian Guo, 2005) between the server and the short message gateway based on the CMPP3.0 (China Mobile Communications Corporation, 2003) protocol of China Mobile which includes CMPPAPI.dll and MFC40.dll (Qiang Ji, 2004). The control consists of the following:

SMSArrive Event: Handles the reception of messages from the short message gateway.

AffirmSMS Event: Handles the status of short messages from the short message gateway.

SMSStart Method: Establishes communications with the short message gateway.

EmitMsg Method: Sends short messages to the short message gateway.

Sconn Method: Reports the status of communications with the short message gateway.

\section{CONCLUSION}

Today the system has successfully been installed in the branch of the China Mobile of Fuzhou City in Jiangxi Province and its application has been extended to every school. The system has been stable for a year. It can send to parents the information whether their children have gotten to school safely in a very short time and can receive short messages from parents without any error. It has been received good feedback from pupils, parents, schools and the branch of the China Mobile of Fuzhou City.

\section{REFERENCES}

Jinhua Tang, Jian Cao. Information Service Platform Based on Short Message. Computer Engineering, 2004.12:238-240

Ruhong Gong, Bin Tang, Renbo Wang. Application of Campus IC Card in Wireless Communication Field, Journal of Henan University of Science \& Technology (Natural Science), 2004, 4:55-57 
Wei Chen, Caopeng Yi, Peixiao Luo. The Implement of SMS Value-Added Applications Platform, Microelectronics \& Computer, 2004, 12:121:67-69

Renbo Wang, Gangyong Lin. SMS Controller Using MCU, Science Mosaic, 2004, 10: 7-8

China Mobile Communications Corporation. China Mobile Peer to Peer (CMPP) v3. 3. 3 [EB/OL]. http://104061.playicq.com/1/18435.html, 2003206

Jian Guo, Lijuan Sun. Design and Implementation of Short Message Service System Based on CMPP, Jiangsu communication technology, 2005, 1:26-29

Qiang Ji. SMPP and CMPP interface of SMS application system, Computers and Applied Chemistry, 2002, 6:786-788 